\title{
Path Optimization in 3D Concrete Printing to Minimize Weak Bonds Formation
}

\author{
Fatima AlSakka', Mohammad Hasan Senan¹, Abdallah Abou Yassin¹, Farook Hamzeh* \\ 1 Department of Civil Engineering, Faculty of Engineering and Architecture, American University of Beirut, \\ P.O. Box 11-0236, Riad El-Solh, Beirut 1107 2020, Lebanon \\ *Corresponding author, e-mail: fh35@aub.edu.lb
}

Received: 19 June 2018, Accepted: 06 February 2019, Published online: 23 September 2019

\begin{abstract}
$3 \mathrm{D}$ concrete printing has proven to be a highly favorable construction method in terms of time reduction, cost optimization, architectural flexibility, sustainability, energy use, and others. However, the quality of the final product certainly has a priority over all of these attractive features of the technology. Yet research has given little consideration to investigating the structural integrity of 3D printed concrete structures. Research states that printed structures exhibit sufficient strength as compared to traditionally built structures. Nevertheless, the fact that this strength is sensitive to numerous factors including the machine setup, the printing process, existing conditions (e. g. temperature) and others, should be studied. A major determinant of the reliability and quality of printed structures is the adhesion level between subsequent layers. Poorly adhered concrete surfaces result in weak bonds that in turn reduce rupture strength. The time elapsed between printing successive concrete layers should be bounded to ensure that concrete is flowable enough to adhere to previous layers. For a given concrete mixture design, this time is a function of travel distance and speed. Thus, this research aims at finding the optimum printing path that minimizes the formation of weak bonds without compromising buildability for a given structure and a defined speed. The research employs Discrete Event Simulation to model the printing process for numerous possible travel paths and assess their adequacy by comparing travel time to allowable time limits.
\end{abstract}

Keywords

3D concrete printing, delay time, DES, path optimization, weak bonds

\section{Introduction}

$3 \mathrm{D}$ concrete printing is an additive manufacturing technique that allows an automated transformation of $3 \mathrm{D}$ design models to actual structures. This is achieved through the deposition of concrete layer after layer (El Sakka and Hamzeh, 2017; Rouhana et al., 2014). The major contribution of this automated construction technique is that it allows building low-income structures in a timely manner (Feng and Yuhong, 2014). Moreover, it enables printing any desired sophisticated structure regardless of its architectural complexity. This is due to the ability of adjusting many parameters such as the printer head speed, nozzle selection, speed of concrete flow and concrete properties (Bos et al., 2016). Despite these and other advantages, one might question the structural integrity of a member that is constructed by depositing thin layers of concrete as compared to structural members that are monolithically cast. It is of no doubt that the formation of poor bonds or cold joints reduces the tensile strength of a structure. To what extent, however, are poor bonds formed in $3 \mathrm{D}$ printed structures?

It might be intuitive to think that joints are unavoidable in a layered system. Yet in reality, tensile strength reduction could be avoided through a proper control of the printing process. The tensile strength between printed concrete layers decreases as the printing time gap between layers increases (Le et al., 2012). In general, the mechanical strength of a layered system decreases and the interface between layers becomes more visually identifiable with the increase in the delay between successive layers (Roussel and Cussigh, 2008). The relationship between elapsed time and joint formation is a function of consistency. If the concrete is workable enough, successive concrete layers properly adhere to each other preventing the formation of poor bonds. Consistency decreases with time and thus, time elapsed between successive layer deposition should be limited. Previous results showed that keeping 
the time gap around 15 minutes results in a high tensile bond strength that could reach 1.5 MPa (Le et al., 2012). Note, nevertheless, that the initial consistency of the mix and the rate of consistency loss affect this time gap.

The most challenging aspect of $3 \mathrm{D}$ printing is the presence of trade-offs between various required mix properties. Layers adhesion necessitates an upper limit for the time elapsed between subsequent depositions. Yet an extruded concrete layer must harden enough to support its own weight in addition to the weight of the successive layer. This imposes a lower limit on time delay. This minimum time elapsed between successive layers is dependent on concrete viscosity and is indirectly proportional to the rate at which the yield stress increases (Roussel, 2007). The maximum horizontal velocity, that ensures a minimum time gap below which the binding of successive layers is compromised, can be calculated from the relation between time and thixotropic behavior (Wangler et al., 2016). Thixotropy means an increase in viscosity when concrete is in a state of rest and a decrease in viscosity when concrete is subjected to a constant shear stress (Khayat et al., 2012). A faster viscosity loss leads to a smaller minimum time necessary for concrete layers to develop a sufficient strength but also a smaller maximum time necessary for layers to adhere to each other.

Hence, since the formation of poor bonds is time dependent, it is a function of printing speed and printing tool path (i.e. travel path). This implies that a proper selection of speed is contingent on the selected printing path, or for a given speed, the path should be optimized to satisfy the criteria of adhesion as well as buildability. Thus, the aim of this paper is to find the travel path that minimizes the formation of poor bonds between layers. This is achieved by designing a simulation model, particularly a Discrete Event Simulation model, of the printing process of a defined structure given a fixed speed. The model helps determine the range of acceptable travel paths. Then, out of the acceptable paths, the one that results in the lowest printing duration is selected as the optimum tool path. Finally, the model provides a basis for building similar models for different design layouts.

\section{Methodology}

To achieve the goal of this research, a well-defined methodology was adopted. As a first step, a literature review was conducted to investigate the factors that affect bonds formation between consecutive concrete layers and the relationships between them. After understanding the type of the targeted problem and its elements, Discrete Event Simulation was selected as a modeling technique that fits the research purpose. Discrete Event Simulation is used for systems whose state changes as events occur at discrete time instances. An important assumption states that nothing important takes place between two successive events (Varga, 2001). The 3D concrete printing process was, hence, modeled as a discrete event system using AnyLogic software. Finally, the obtained results were analyzed and compared against adhesion and buildability criteria. These steps are summarized in Fig. 1.

A simple design layout was selected for this study to reduce the complexity of the model. It consists of a square area $(16 \mathrm{~m} \times 16 \mathrm{~m})$ divided into four equal squares. Although actual design layouts are much more complicated than the adopted design, the latter was deemed to have an adequate level of details for the purpose of building the desired model from scratch. The model can be further expanded, and more complex designs can be simulated by following the same logic.

The simulation of the printing process helps determine the optimal travel paths and any printing gap time if needed. This is achieved by designing the model to give the list of all the possible printing paths. The various printing paths as well as the time it takes to print a complete layer are traced in each simulation run and recorded. Then for each path, the total waiting time of a concrete layer after being casted is compared to an upper and a lower allowable time limits to avoid formation of cold joints while maintaining layer buildability. This gives the range of the acceptable traveling paths that satisfy adhesion and buildability.

\section{Model objectives}

The global objectives of designing a Discrete Event Simulation model for the 3D concrete printing process subsume the following:

- "Reveal the apparently simple to be complex" (Epstein, 2008). Selecting a path for printing might sound easy. However, once the criteria required for obtaining a printed structure that meets minimum quality standards, the trade-offs that exist between these criteria, and the numerous possible paths that can be taken are determined, this task becomes difficult.

- Identify the optimum travel path that prevents the formation of poor bonds between subsequent concrete layers and, at the same time, allows concrete layers to harden enough to support successive layers. 


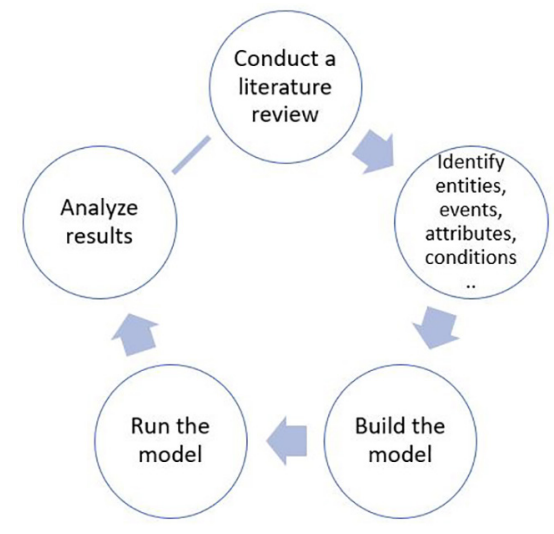

Fig. 1 Research plan

- Establish a basis for designing future, more complex designs by providing the logic for building such models.

These objectives are to be achieved and further analyzed throughout this study.

\section{Model development}

\subsection{Conceptual level}

In the proposed model, the $3 \mathrm{D}$ concrete printer is modeled as an entity with different states, "Printing" or "Passing". The structure is modeled as consisting of twelve vertical and horizontal links along which concrete must be deposited and eight diagonals along which concrete should not be extruded (Refer to Fig. 2). Each link is considered an entity of which the state is either "Printed" or "Not Printed". An event takes place when the printer crosses a link. If the link is "Not printed", the printer state becomes "Printing" and the link becomes "Printed". If the link is "Printed", the state of the printer changes to "Passing".

As shown in Fig. 2, nine points are marked on the layout. The printer can start at any of the corner points $(1,3,7,9)$ or the middle point $(5)$. When the printer is at

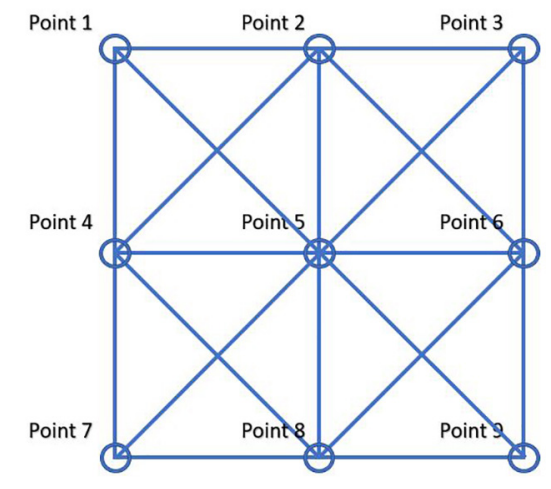

Fig. 2 Design layout a certain point, it can travel to any one of the neighboring points except for the last point it crossed. As previously mentioned, concrete is to be laid across only twelve links. When all these twelve links are printed, the printing cycle stops, and a new cycle is initiated. The model provides all the possible sequences of traveled links that lead to printing a complete concrete layer.

\subsection{Specification level}

Obtaining a sufficiently adhered concrete layers is essential for building structurally sound members. A concrete layer should remain enough workable before a new layer is deposited. Otherwise, concrete layers would become too stiff to intermix with subsequent layers and colds joints would result. This implies that bonding between layers would be weak and, thus, tensile strength would be low, possibly leading to structural failure. Hence, the time elapsed from the point at which the first layer is printed to the point at which the next layer is printed should be limited. An upper limit for this time value is expressed in Eq. (1).

$t_{h, \max }=\frac{\sqrt{\frac{(\rho g h)^{2}}{12}+\left(\frac{2 \mu_{p} V}{h}\right)^{2}}}{A_{\text {thix }}}$

where $V$ is the horizontal linear velocity, $\rho$ is the density of concrete, $\mu_{p}$ is the plastic viscosity, $h$ is layer thickness, $g$ is the gravitational acceleration, $A_{\text {thix }}$ is the rate at which yield stress increases.

For this study, these terms are given the following values $V=20 \mathrm{~cm} / \mathrm{s} ; \rho=2400 \mathrm{~kg} / \mathrm{m}^{3} ; \mu_{p}=50$ Pa.s; $h=3 \mathrm{~cm} ; g=9.81 \mathrm{~N} / \mathrm{Kg} ; A_{\text {thix }}=2 \mathrm{~Pa} / \mathrm{s}$.

The corresponding upper limit is:

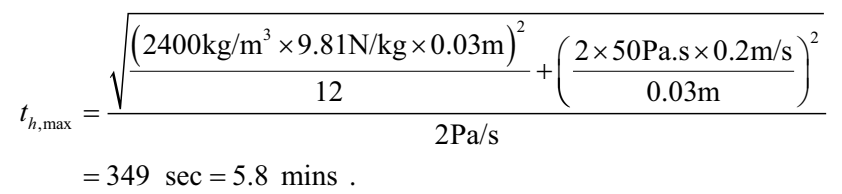

This means that time needed to produce a concrete layer should not exceed 5.8 mins. On the other hand, if concrete layers are too workable, they will not be able to support their own weight or the weight of subsequent layers. This means that buildability will be lost. Thus, the travel rate should not be too high to allow concrete to develop the sufficient strength needed for buildability. The minimum time required to create a new layer is expressed in Eq. (2). 


$$
t_{h, \min }=\frac{\rho g h}{\sqrt{3} A_{t h i x}}
$$

In this study and given the same previously defined terms' values, the minimum time necessary for producing a concrete layer is

$$
\begin{aligned}
t_{h, \text { min }} & =\frac{2400 \mathrm{~kg} / \mathrm{m}^{3} \times 9.81 \mathrm{~N} / \mathrm{kg} \times 0.03 \mathrm{~m}}{2 \sqrt{3}} \\
& =204 \mathrm{sec}=3.4 \mathrm{mins} .
\end{aligned}
$$

Therefore, the time needed to extrude a concrete layer to prevent poor bonds formation while maintaining buildability should be limited between 3.4 and 5.8 mins. Any path of which the printing duration falls outside this range is rejected.

\subsection{Computational level}

At this level, a computational model is developed. Even though the concept seems very simple, Fig. 3 shows how complex the situation actually is. This verifies one of the modeling objectives which consists of revealing the possible complexity of a process that appears to be simple.

The printer, which flows through the system, is generated from a source. The printer enters a queue before it moves to one of five points. The choice of the destination point is made random by using selectOutput 5 command from AnyLogic library and assigning equal probabilities to different links. Points are modeled as queues and passing/printing links are modeled as delays. The delays are assigned durations computed by dividing the travel distance (i.e. link length) by the horizontal linear velocity. Each time the printer arrives to a certain queue,

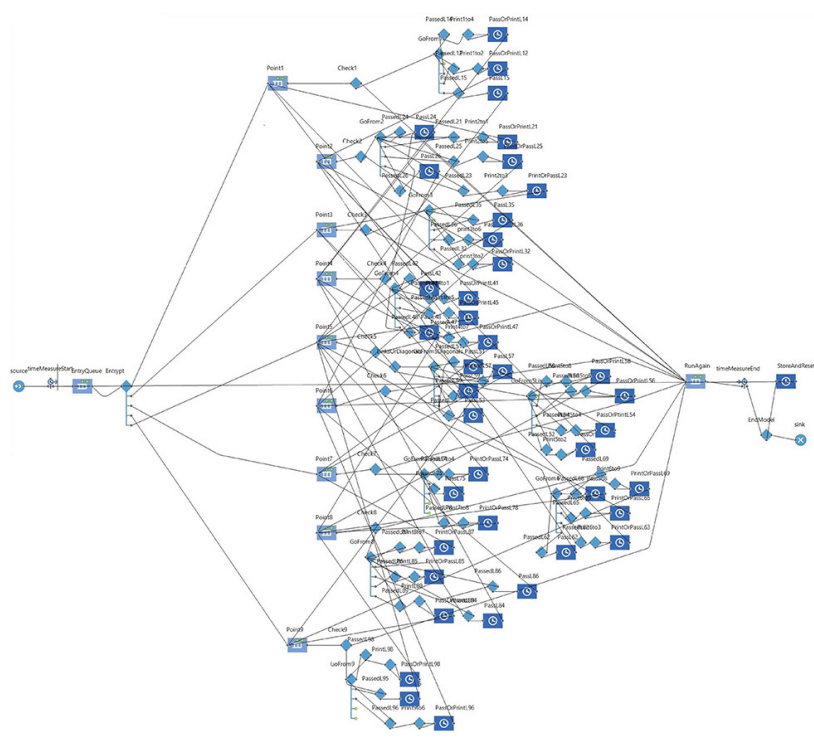

a certain link is chosen also using selectOutput command and the type of the activity/delay, "Passing" or "Printing", is determined based on the state of the link, "Printed" or "Not Printed". Once a total of twelve vertical and horizontal links become printed, everything is restored to their initial conditions and the printer repeats the same process to record a new path.

The model is designed to allow the user to set the total numbers of possible paths (runs) needed. Once this number is obtained, the printer is disposed into the sink and the simulation run stops.

\section{Simulation results}

\subsection{Paths results}

Running the model results in an excel file comprising the printing duration and the path taken for each printing cycle/run. Accepted paths are marked with a green check sign. A sample of the results is illustrated in Fig. 4. In the results, the links are denoted in a specific format (start, end) to indicate the direction in which the printer is travelling. For instance, L32 indicates that the printer is travelling from point 3 to point 2 .

The model was run a total of 10,000 times, of which 9,703 paths were not accepted since their printing duration was outside the acceptable time range (3.4 to 5.8 mins). These paths were rejected since their total durations result either in poor bonding between successive layers or in distorted layers' pattern.

297 paths had durations that lie within the acceptable time range. The shortest acceptable path had a time of 4.8 mins ( $288 \mathrm{sec})$, and the longest acceptable path had a

Fig. 3 Discrete Event Model using AnyLogic 


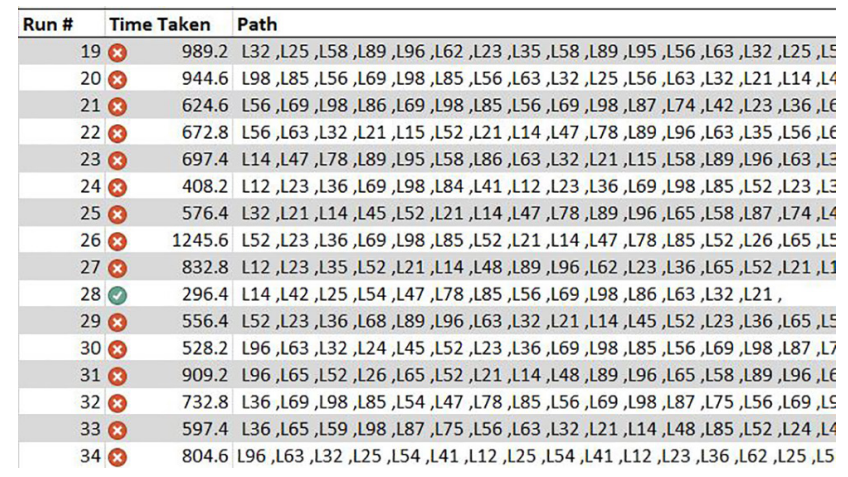

Fig. 4 Run results

time of 5.7 mins (344 sec). However, the longest rejected path had a total time of $45 \mathrm{mins}(2704 \mathrm{sec})$. It is important to note that even though this value is not realistic, it shows up in the model since the printer moves randomly across the links. Such behavior can lead to passing the same links several times redundantly. These cases are disregarded anyway since they do not satisfy the requirements. The graph shown in Fig. 5 summarizes the results of the 10,000 runs sorted from the lowest accepted times (grey color) to the largest rejected times (orange color).

Note that for this design and the chosen horizontal velocity, all the paths fulfilled buildability requirement since the lowest duration needed to print a complete layer was $288.2 \mathrm{sec}$, which is greater than the minimum time for layer reduction $(204 \mathrm{sec})$. Thus, the criticality of a certain criterion related to $3 \mathrm{D}$ concrete printing is contingent on the design layout and machine setup. In this study for instance, adhesion criterion is more critical than constructability criterion.

Fig. 6 shows the distribution of the obtained results. The distribution shows that most of the resulting paths do not comply with the accepted time range. It is important to note that this data reflects the results of a relatively simple and symmetrical structure. However, if a more complex structure was modelled, the distribution of the results may be different, and most probably will be more skewed to the right.

\subsection{Optimum paths}

Since 297 out of 10,000 paths fall within the acceptable time range, the paths with the shortest duration should be selected to optimize time and cost. Although other paths with longer durations are acceptable in terms of adhesion, they comprise some movements that are deemed unnecessary and wasteful. Hence, they are disregarded. In the obtained results, 13 paths had the shortest time of 4.8 mins. Fig. 7 shows these paths in more details.

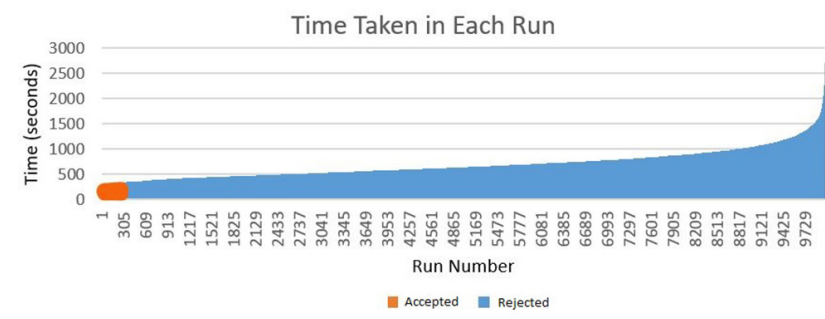

Fig. 5 Summary of results

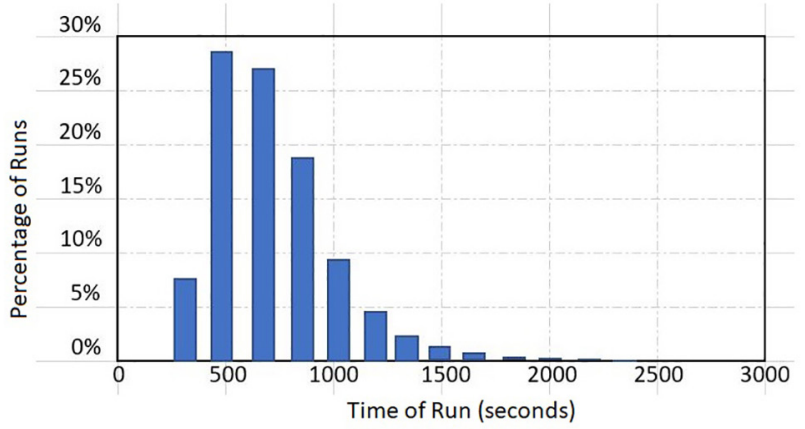

Fig. 6 Distribution of results

\begin{tabular}{|c|c|c|}
\hline Run \# & Time Taken & Path \\
\hline $2210 \mathrm{C}$ & 288 & 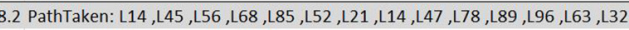 \\
\hline 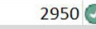 & 288. & 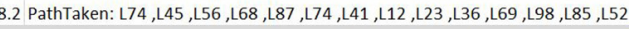 \\
\hline 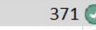 & 288 & 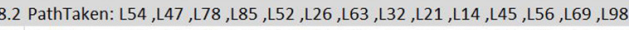 \\
\hline 3069 e & 288. & 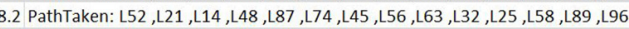 \\
\hline 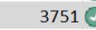 & 288. & 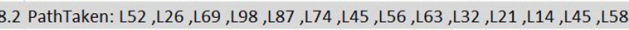 \\
\hline 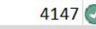 & 288. & 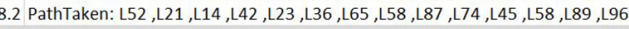 \\
\hline 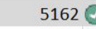 & 288 & 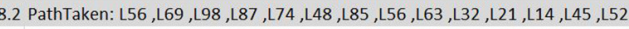 \\
\hline 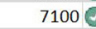 & 288. & 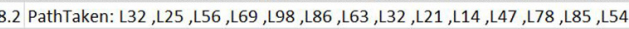 \\
\hline 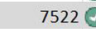 & 288. & 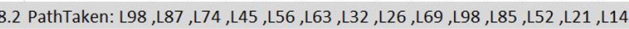 \\
\hline 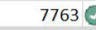 & 288. & 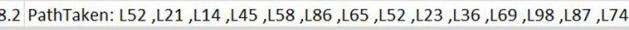 \\
\hline $8119 e$ & 288. & 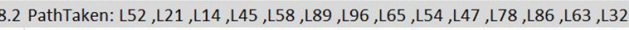 \\
\hline 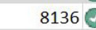 & 288. & 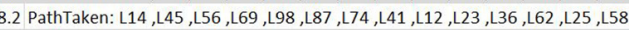 \\
\hline 9809 e & 288. & 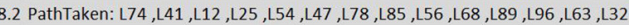 \\
\hline
\end{tabular}

Fig. 7 Shortest paths

From these results, it can be observed that all these paths only have one diagonal 'passing' link. The results show that most of the paths started from the center point (point 5). It is important to note that the symmetry of the structure used in this study leads to obtaining symmetrical paths with equal printing durations. Were the model run infinitely, all these paths would have resulted, and the model would have been verified. If a more complex structure was modelled and simulated, it is expected that much fewer paths will have the same shortest durations. For a more complex geometry, the model might not even find any acceptable path under the prevailing conditions. In that case, manipulating the printing speed, the extrusion rate, the thickness of concrete layers, the mixture design is deemed necessary.

One of these optimum paths is traced in Fig. 8. The printer starts at the black node, travels through the black links then through the orange links and stops at the orange node. Note that the printer does not extrude concrete along the dotted lines. 


\section{Conclusion}

The formation of cold joints in 3D printed concrete structures and their impact on durability is yet an open research question. This paper aimed at establishing a systematic methodology to optimize 3D printing paths for minimum poor bonds formation. Moreover, a Discrete Event Model was developed using AnyLogic software to simulate the 3D printing of a simple symmetrical structure. The model was run 10,000 times and 297 paths were identified to satisfy a required time range criterion. The time range calculated is dependent on the properties of the concrete mix and the horizontal velocity of the printer. The lower limit of the time range will allow the concrete layers to harden enough to support successive layers while the upper time limit will limit the formation of poor bonds between subsequent concrete layers. In the produced model, all the paths fulfilled the buildability requirement. On the other hand, of the 297 accepted runs, only 13 paths were chosen to have the optimal (shortest) travel distance and time. The data obtained from the simulation, which consisted of the duration and the exact route taken by the printer on each path, can be exported to a spreadsheet for further analysis. The model was built as a proof of concept and should act as a basis

\section{References}

Bos, F., Wolfs, R., Ahmed, Z., Salet, T. (2016) "Additive manufacturing of concrete in construction: Potentials and challenges of $3 \mathrm{D}$ concrete printing", Virtual and Physical Prototyping, 11(3), pp. 209-225. https://doi.org/10.1080/17452759.2016.1209867

El Sakka, F., Hamzeh, F. R. (2017) "3D Concrete Printing in the Service of Lean Construction", In: 25th Annual Conference of the International Group for Lean Construction, Heraklion, Crete, Greece, pp. 781-788. https://doi.org/10.24928/2017/0246

Epstein, J. M. (2008) "Why Model?", Journal of Artificial Societies and Social Simulation, 11(4), p. 12. [online] Available at: http://jasss. soc.surrey.ac.uk/11/4/12.html [Accessed: 01 June 2018]

Feng, L., Yuhong, L. (2014) "Study on the Status Quo and Problems of 3D Printed Buildings in China", Global Journal of HumanSocial Science Research, 14(5), pp. 1-4. [online] Available at: https://globaljournals.org/GJHSS_Volume14/E-Journal_GJHSS_ (H)_Vol_14_Issue_5.pdf[Accessed: 01 June 2018]

Khayat, K. H., Omran, A. F., Megld, W. A. (2012) "Evaluation of thixotropy of self-consolidating concrete and influence on concrete performance", In: 3rd Iberian Congress on Self Compacting Concrete, Madrid, Spain, pp. 3-16.

Le, T. T., Austin, S. A., Lim, S., Buswell, R. A., Law, R., Gibb, A. G. F., Thorpe, T. (2012) "Hardened properties of high-performance printing concrete", Cement and Concrete Research, 42(3), pp. 558-566. https://doi.org/10.1016/j.cemconres.2011.12.003

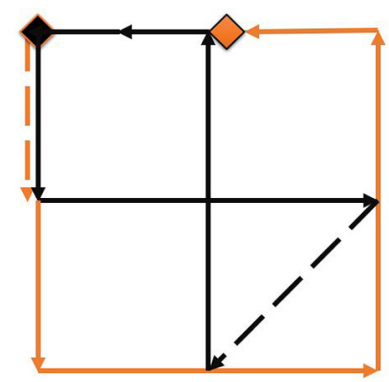

Fig. 8 One optimum path

for developing new models to optimize 3D printing paths for more complex structures. Following the same logic from the built model, the achieved path optimization will aid in forming more sound and durable concrete structures with the least possible waste. Further experimentation and research should be made to verify and validate the results of the model. This can be done by printing identical 3D concrete structures using different accepted paths obtained from the model and then testing the bond strengths in each one of them. Further research could also focus on producing an Agent Based Model that is more generic and will allow the end user to quickly import a CAD drawing and run the simulation to get the optimized path.

Rouhana, C. M., Aoun, M. S., Faek, F. S., Eljazzar, M. S., Hamzeh F. R. (2014) "The Reduction of Construction Duration by Implementing Contour Crafting (3D Printing)", In: 22nd Annual Conference of the International Group for Lean Construction, Oslo, Norway, pp. 1031-1042. [online] Available at: https://pdfs.semanticscholar.org/49a8/765f19e6fd607f261a010880d74984b6c2ba.pdf [Accessed: 01 June 2018]

Roussel, N. (2007) "Rheology of fresh concrete: From measurements to predictions of casting processes", Materials and Structures, 40(10), pp. 1001-1012. https://doi.org/10.1617/s11527-007-9313-2

Roussel, N., Cussigh, F. (2008) "Distinct-layer casting of SCC: The mechanical consequences of thixotropy", Cement and Concrete Research, 38(5), pp. 624-632.

https://doi.org/10.1016/j.cemconres.2007.09.023

Varga, A. (2005) "OMNeT++ Discrete Event Simulation System, Version 3.2, UserManual", [online] Available at: http:/www2.ensc.sfu.ca/ people/faculty/ljilja/ENSC835/News/Presentations/OMNeT++/ usman.pdf [Accessed: 01 June 2018]

Wangler, T., Lloret, E., Reiter, L., Hack, N., Gramazio, F., Kohler, M., Bernhard, M., Dillenburger, B., Buchli, J., Roussel, N., Flatt, R. (2016) "Digital concrete: Opportunities and challenges", RILEM Technical Letters, 1, pp. 67-75. https://doi.org/10.21809/rilemtechlett.2016.16 